\title{
Brain-Computer Interface: Next Generation Thought Controlled Distributed Video Game Development Platform
}

\author{
Payam Aghaei Pour, Tauseef Gulrez, Omar AlZoubi, Gaetano Gargiulo and Rafael A. Calvo
}

\begin{abstract}
In this paper we present a system that uses the human ability to control a video game on a mobile device using electroencephalographic (EEG) Mu rhythms. The signals were obtained using a specially designed electrode cap and equipment, and sent through a Bluetooth connection to a PC that processes it in real time. The signal was then mapped onto two control signals and sent through wireless connection to a mobile gaming device BreakOut ${ }^{-}$. We have also investigated the human's ability to play the video game by manipulating neuronal motor cortex activity in the presence of a visual feedback environment. The participants played the video game by using their thoughts only with up to $80 \%$ accuracy over controlling the target.
\end{abstract}

\section{INTRODUCTION}

Brain-Computer Interface (BCI) technology [1], [2], [3], [4], [5] is a new and fast evolving field that seeks direct interaction between the human neural system and machines, aiming to augment human capabilities by enabling people (especially disabled) to communicate and control devices by mere "thinking" or expressing intent.

The increasing success of BCI systems is partially due to a better understanding of the dynamics of brain oscillations that generate EEG signals. In the brain, networks of neurons form feedback loops responsible for the oscillatory activity recorded in the EEG. Normally the frequency of such oscillations becomes slower with increased synchronization. Sensorimotor activity such as motor movements or motor imagery (e.g. imagining hand/feet movement) changes the oscillatory patterns resulting in amplitude suppression (Event Related Desynchronization - ERD) or amplitude enhancement (Event Related Synchronization - ERS) on the Rolandic mu rhythm $(7-13 \mathrm{~Hz})$ and the central beta rhythms above $13 \mathrm{~Hz}$.

A second reason for the increased interest in BCI is the improved resolution and lower cost of recording equipment. Current research [6], [7] aims at developing systems that use dry electrodes instead of the cumbersome golden or $\mathrm{Ag} / \mathrm{AgCl}$ electrodes, that require gel, glue and skin preparation. This technology, together with making the devices more mobile, will allow BCI systems to be viable for gaming. The Emotiv gaming device [8], recently announced, is believed to use electrophysiological and physical (a gyroscope) signals for control. This system has not been described in the academic literature.

P. Aghaei Pour is with ICT Internetworking at The Royal Institute of Technology, Stockholm/Sweden and a visiting scholar at the School of Electrical and Information Engineering. The University of Sydney, Australia.

T. Gulrez, O. AlZoubi, G. Gargiulo and R.A. Calvo are with School of Electrical and Information Engineering, The University of Sydney, Australia. email:rafalee.usyd.edu.au

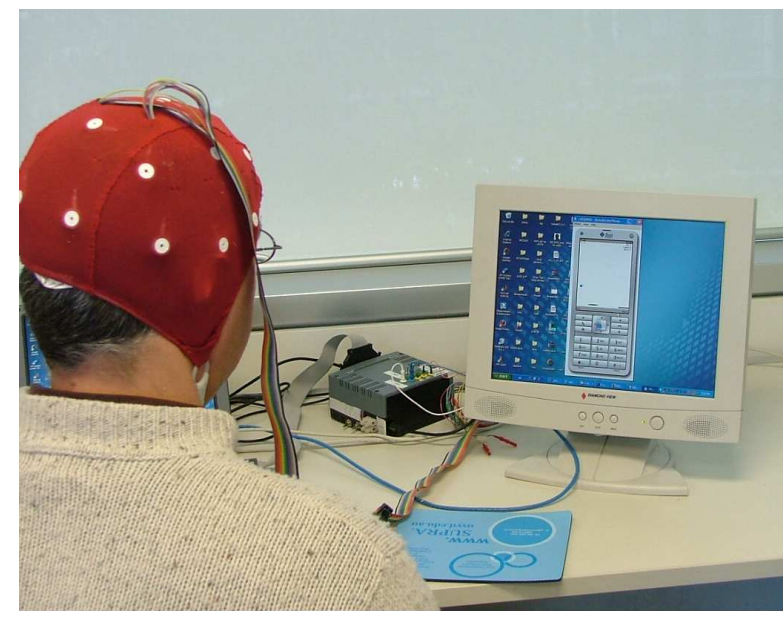

Fig. 1. An EEG based brain data recording/controlling system for playing video games.

Third, old generation EEG-based BCI had a limited communication capacity and it looked very unlikely to be suitable for control [4]. Based on signal acquisition methods, BCI can be categorized by whether it uses non-invasive (e.g. EEG) or invasive (e.g. intracortical) techniques. Progress on the signal processing and the classification algorithms has increased the communication capacity to a point that real applications are now feasible.

A key to the success of any BCI system is the extraction of a set of optimum features that reduce the high dimensionality of a multi-channel EEG signal. These features are then used to train a classifier that takes the signals features and produces either a real-valued quantity or a discrete classification [9]. When many channels are used Principal Component Analysis (PCA) [10] can decompose the multichannel signal into a reduced number of features that preserve the maximum explained variance. Other techniques such as Independent Component Analysis (ICA) [11] and Maximum Signal Fraction Analysis (SFA) [12] has also been used in BCI systems. In each technique the resulting features optimize a different cost function and have different properties. ICA maximizes the information carried by the signals and maximum SFA optimizes the signal to noise ratio. Early evaluation of classification techniques for EEG signals [13] showed that Tree-based Neural Networks (TBNN) and tree-based radial basis function networks (TB-RBF) can classify hypnograms with similar accuracy to clinical experts. More recently, a taxonomy of the feature extraction and classification techniques used in BCI was documented in [14]. A 
further review of numerous techniques used for classification of EEG signals [9] showed that Support Vector Machines as well as combination of classifiers are particularly efficient for synchronous BCI. Other studies showed that classifier performance depends on factors such as, the experimental set-up, the montage and the user task [15].

One of the difficulties encountered in such reviews is that it is hard to compare the classifiers unless classifiers are tested with the same context, feature extraction methods and experimental protocols. In order to overcome this problem a number of research groups use BCI frameworks such as BCI2000 [16] that provide a structured approach for testing feature selection and classification techniques. Even then the comparison for online BCI is hard since the computational requirements become an issue. For this reason most classifiers used in real time scenarios are relatively simple, and are always run in a computer with a fast CPU, not on mobile devices.

After the features have been extracted the classification algorithm generate control signals based on the signal features. These commands may be continuous or discrete. They should be as independent as possible, for example horizontal movement should not depend on vertical movement [4]. The success of a classification algorithm is determined by the appropriateness of its selection of signal features and much research has been done to determine the most effective features in the brain signals [17], [18]. These algorithms may adapt to each user on three levels. First, when a user uses the BCI for the first time, the algorithm adapts to the user's signal features. Second, brain signals may be changed based on the different factors such as, time of day, hormonal levels, immediate environment, and illness. To keep the algorithm adapted to the condition, periodic online adjustments are needed to reduce the impact of those spontaneous variations. The third level of adaptation engages the adaptive capabilities of the brain [19], where the brain modifies or remap the signal's features to improve BCI operation [20], [21].

This paper reports on the implementation of a wireless BCI system used to play a simple game on a mobile device. The paper is organized so that, Section II throws light on the basic methodology to conduct BCI for video game experiments and a brief description of EEG data recording and analysis necessary to control the video game. Section III describes the distributed architecture of our BCI-Video game platform. This paper concludes in section $\mathrm{V}$.

\section{EVALUATION}

\section{A. Participants}

The EEG brain signals data were recorded from four male subjects at the University of Sydney. The selected participants were between 20-40 years old with no experience using the game, and only little experience using BCI systems.

\section{B. EEG Data Recording}

Participants were fitted with a head-mounted EEG cap as shown in Figure 1. Signals were acquired from three

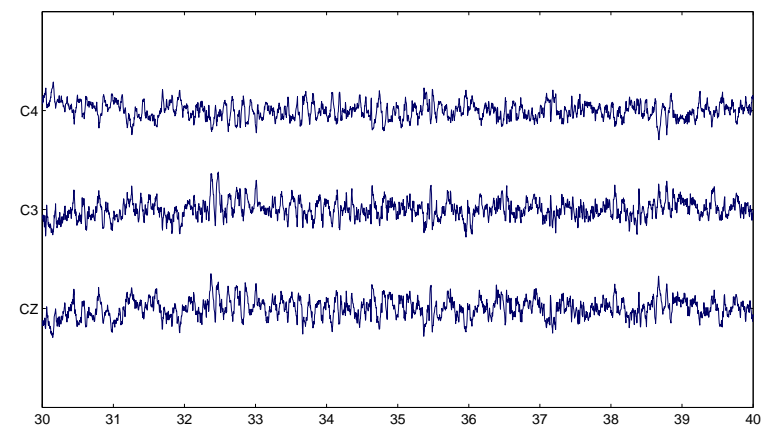

(a) Raw EEG data recording using three electrodes over the motor cortex area of a participant. The three electrodes $\mathrm{C} 3, \mathrm{C} 4, \mathrm{Cz}$ data are plotted in a window of 10 seconds to analyze the mu-rhythm activity.

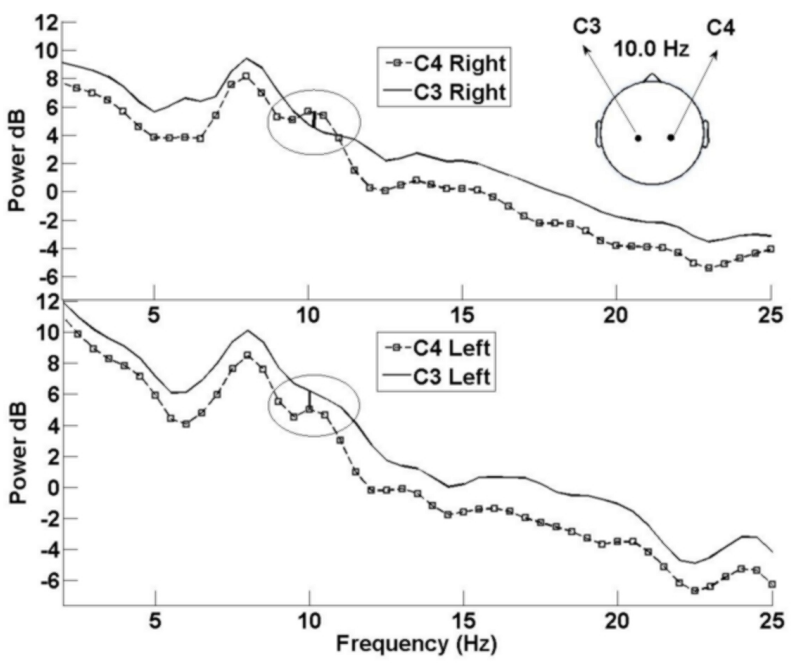

(b) The figure shows spectral power during imagery hand movements at electrode locations $\mathrm{C} 3$, and $\mathrm{C} 4$.

Fig. 2. Participant's Motor Cortex EEG signals responsible to control the BreakOut $^{-}$on the PDA device.

electrode sites $\mathrm{C} 3, \mathrm{C} 4 \& \mathrm{Cz}$ according to the international $10 / 20$ system. A unipolar signal (as shown in Figure 2) was recorded using the left ear as a reference and the right ear as the ground. EEG activity was recorded at a sampling rate of $256 \mathrm{~Hz}$ using a bandpass filter of $0.1-100 \mathrm{~Hz}$ and an amplification ranging from 10,000 to 20,000 (depending on the subject's baseline mu power).

\section{Subject Training to control the Video Game}

Participants sat in a comfortable chair with armrests, and asked to look straight ahead at a computer monitor that displayed a high-resolution BreakOut ${ }^{-}$video game, as shown in Figure 1. To familiarize participants with the control scheme of the video game an initial training period was conducted, 
where subjects were asked to explore the game by pressing the keys on the keyboard to move the BreakOut ${ }^{-}$paddle to left or right. At the end of the initial training period, participants started their control of the BreakOut ${ }^{-}$game, subjects were instructed not to touch the keyboard, but to attempt to move the BreakOut ${ }^{-}$paddle to the right or left by imagining right/left hand movement. The participant's data were recorded upon the completion of their session. Muscle artifact were not removed from the signal, so it was important that the subjects move the least possible. In BCI applications targeted to locked-in patients muscle artifact should be removed.

The classification task is done through the linear classifier included in BCI2000, this classifier does not learn from the data and does not adapt, as other classifiers do [9], [15]. The classifier produces a control signal, the cursor is moved on the screen by a linear function of this control signal. This approach was evaluated by [22] defining $\Delta V$ as the cursor movement, a defined number of control signals of past trials is used to compute the mean a, the inverse of the standard deviation is set as the gain $\mathrm{b}$, and $\mathrm{S}$ is the control signal, the function that we use to determine each cursor movement is $\Delta V=b(S-a)$.

Right and left movements were controlled by using a differential $\mathrm{Mu}$ over both brain hemispheres as the bases for the classification, that is the $\mathrm{Mu}$ power difference over electrode locations C4, C3 $(C 4-C 3)$. Figure 2(b) shows a decrease in power around $10 \mathrm{~Hz}$ at $\mathrm{C} 3$ during right hand imagery movement, the bottom figure on other hand shows a power decrease around the same frequency level at $10 \mathrm{~Hz}$ at $\mathrm{C} 4$ during left hand imagery movement. This conforms with the expected behavior of the Mu rhythmic activity during hand imagery movement at this frequency level.

\section{A Distributed Architecture Design}

Distributed applications commonly require the sharing of computation and storage between their components. In this project, we have used a distributed architecture for playing video games with the brain signals. While mobility has been added by introducing a wireless EEG acquisition machine and wireless application device, performance is kept the same as previous BCI solutions. This has been done by keeping the entire computational intensive tasks (e.g. machine learning and signal processing) on the stationary base computer like laptop. Our distributed architecture is illustrated in Figure 3.

\section{A. Bluetooth Connection}

The EEG signals are acquired by the Penso system [7] that allows for wet or dry electrodes to be used (we used wet electrodes for this study). The Penso recording equipment is connected to a Tablet PC through a Bluetooth interface. The BluesenseAD was selected as a Bluetooth [23], [24], [25], [26] transceiver for its compliance with the safety standards required in medical applications, it has 8 analog to digital channels, it is able to sample data at $4000 \mathrm{~Hz}$ for a single active channel to $150 \mathrm{~Hz}$ for an 8 activated channels, and has low power consumption. Bluesense packets have
TABLE I

BluesenseAd PaCKet Structure

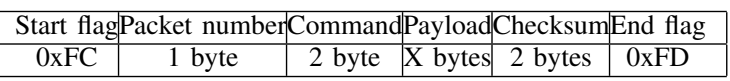

a similar structure to the AT commands (which are often used to establish modem connections). Table-I shows the format of the packet, where Start and End flag determine the boundary of the packet so there is no limitation in the length of the packet. Packet number is used to detect packet loss and each side should pick a separate number and increase it consecutively. The Command field describes which command has been sent or received and based on the command, each side can decide what has to do next. Payload could be any data such as sampled data or further information related to the requested command. Checksum is calculated over the entire packet except the Start Flag, End flag and of course Checksum field.

After the sampled data is transferred over an established Bluetooth connection to the tablet PC, BCI2000 is used for signal processing and classification to produce a control signal, which is then sent wirelessly to the PDA.

\section{B. Signal Acquisition and Processing}

BCI2000 [27] provides a flexible general-purpose platform that facilitates the evaluation, comparison, and combination of brain signals, translation algorithms, applications, and operating protocols. BCI2000 can reduce the time, effort, and expense of testing new designs and also provide a standardized data format for offline analysis [16]. As shown in Figure (3-1), EEG signals were recorded with the BCI2000 as the platform for signal acquisition and processing. Signals were read into a base-computer that determined the near realtime level of differential mu power and relayed it to a second computer running the video game as described in the sectionIII-D. The following are the BCI2000's modules [16] used towards the video game application:

1) Source Module: The recording system we used [7] has a Bluetooth transceiver that allows 25 meters of operating range, a data rate of up to $115,200 \mathrm{bit} / \mathrm{s}$ in 8 channels sampled at 12 bits with a maximum frequency threshold of $4 \mathrm{KHz}$. We also used a different version of the same system with a data acquisition module connected to National Instruments DAQ card-1200, a different BCI2000 driver was implemented for this amplifier, both systems had similar performances. The source module digitizes and stores brain signals and passes them to the signal processing module. It consists of a data acquisition and a data storage component. We extended the module with a driver specific to our Bluetooth device. Data storage, stores the acquired brain signals in a standardized data file format.

2) Signal Processing Module: The signal processing module converts the raw signals from the brain into meaningful signals responsible to control an external device or application. This conversion has two components: feature extraction and feature translation. Features were derived 


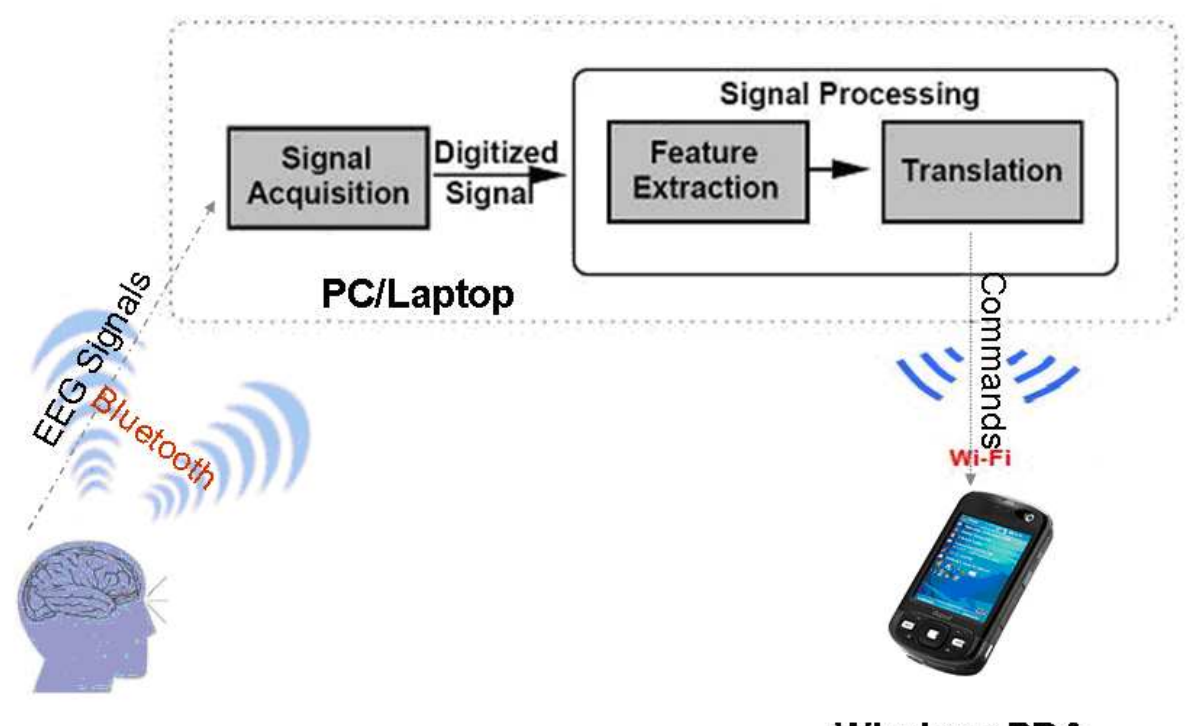

Wireless PDA

Fig. 3. Proposed Distributed Architecture for Brain-Computer Interface Design for Playing Video Games.

from a signal of window length of $0.5 \mathrm{~s}$ using an autoregressive [28], [29] filter of order (16) which enabled us to estimate the spectral amplitude values of the signal, 11 bins of equal size for frequencies between $0 \mathrm{~Hz}$ and $31.5 \mathrm{~Hz}$ were obtained. A subset of these evaluations that correspond to a selected frequency levels then were passed into the linear classification algorithm; the linear classifier computes a projection of the high dimensional signal feature space onto a low dimensional classification space. In other words, each dimension of the classification space can be represented as a linear combination of the signal features. The output of the linear classifier is then normalized with respect to mean and variance to produce the control signal which is then used to determine the cursor movement. The filters in BCI2000 [27] are completely independent, hence, they can be combined or interchanged without any system alteration.

\section{Client/Server Application Module}

BCI2000 provides a way to directly communicate with an external device through UDP. However UDP is not reliable and does not provide an ease of communication towards the Real-time Java Mobile applications. In lieu. of that, a gaming server application has been created using Java NetBeans IDE 6.0 technology running under the WindowsXP environment on the host HP-Compaq TC 4400 tablet PC having 1.0 GB of RAM and Intel dual core $1.8 \mathrm{GHz}$ of processing speed. The NetBeans IDE is an Open-Source Integrated Development Environment (IDE) for software development, which provides all the necessary tools needed to create professional cross-platform desktop, enterprise, Web and mobile applications. The client side Java Mobile gaming application 'Breakout ${ }^{-}$', has been created and can run dually on desktop PC under SUN-Microsystems's emulator environment or on mobile devices such as PDAs and mobile phones. The client/server application module sent through the performance measure statistics of the participants while playing the 'Breakout ${ }^{-}$' using the brain signals, which are further analyzed in the section-IV of this paper.

1) User Application Module: The user application module receives control signals from signal processing and uses them to drive an application. This module is augmented with connector component which enables BCI2000 to transmit all the control signals over user defined IP address and UDP port to the gaming server that manages the game running on the mobile device.

2) Operator Module: The operator module defines the system parameters (e.g., the trial length in a specific application or a specific signal processing variable). In addition, operator can display information (e.g., a text message or a signal graph) sent to it from any other module. This allows a user to view real-time information about online events (e.g., display of unprocessed brain signals).

\section{BreakOut ${ }^{-}$Video Game}

We have adapted the popular BreakOut game to a simpler version, more appropriate for the BCI task. Bricks have been removed to reduce the nondeterministic matter of ball landing point but still it had a kind of strategy and it might result in failure due to the bouncing ball. In a beginners mode, a green bar at the bottom has been added to indicate the landing point of the Ball, so the user should not think about anything else except moving the bar to the target. In an expert mode this green bar is eliminated. In Figure 4 the picture of the new Breakout layout is depicted running within the emulator. The game was tested using an HTC phone running Windows Mobile 5, and a HP PDA hx2400.

The output of signal processing module sends device commands that can be used to control any device. For this project we developed the Java game as a prototype application for PDA. The wi-fi has been used as a wireless 


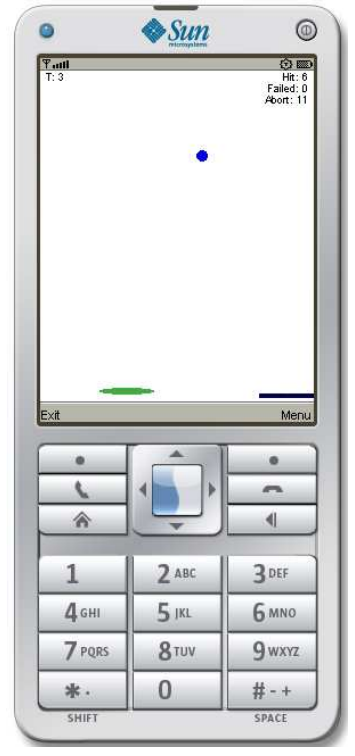

Fig. 4. BreakOut ${ }^{-}$Video Game, developed under Java NetBeans can easily be ported on any mobile (PDA) device.

connection between PDA and the Tablet PC. TCP or UDP can be used as a protocol to exchange data between PDA and PC. Since some Java-enabled PDAs do not support UDP, both TCP and UDP have been developed.

The main advantage of this architecture is keeping the gaming device mobile while having sufficient computational power for machine learning or classification algorithms. We substituted wireless connection instead of the wires used to go from EEG electrodes to computer which has provided more freedom for EEG cap wearer. This architecture takes us one step closer to wearable BCI system.

\section{RESUlts}

Table-II shows, the four participant's statistics, the EEG control-level across all the participants not varied too widely over the first 3-4 sessions. Every participant achieved above random measure of control i.e. ( $>50 \%$ for everyone with the max $89 \%$ of accuracy): there was no significant change in the accuracy across all the four sessions. Table-II also indicates that, for this relatively small number of participants, high accuracy $(>90 \%)$ ususally takes several weeks or months to develop and that subjects vary greatly in their learning rates. This is consistent with the hypothesis that subjects learned to produce diffrent levels of power over each brain-hemisphere and, thus, produce 'low' mu very quickly and were able to maintain that level of control from the first to the last training session. One participant was able to maintain a control level higher than his initial baseline threshold for $77 \%$ of the time during the first training session period. This level of control improved to $100 \%$ on the second and fourth training session and maintained at $80 \%$ for the remainder of the experiment.

\section{Conclusions}

In this study we have developed a distributed framework for controlling the BreakOut $^{-}$video game through brain signals. The participants learn to control their mu rhythms, especially when this learning involves controlling mu levels over the sensorimotor cortex in each hemisphere. They are able to maintain that level of control across 5-6 training sessions. The distributed architecture of our gaming environment has two positive effects on the whole learning process. Firstly, the brain data can be transmitted without the ribbon cable, allowing our participants to sit in a comfortable natural posture and concentrate on the video game control without any position specific distractions. Secondly, having data processed on the PC separate to the video game console gives low delays in the control signals, whereas the computation power of signal processing and feature extraction becomes exponentially higher.

This study has raised a number of open questions for future work. Firstly the adequacy of number of trials that are needed to map the brain signals to produce similar versus different levels of mu across each hemisphere. In a similar way we could also answer the question of whether or not there are limits to the level of $\mathrm{Mu}$ control that can be produced? Secondly, do previous experiences such as video games or sports-manship affect the control levels. Unfortunately, the sample size presented in this paper was too small to allow analysis/out of scope of this paper.

The video game environment also plays a vital role in motor learning and control process, hence should be examined more thoroughly to determine whether better visualization of the signal could improve training. We are in the process of developing a 3D-Virtual Reality (VR) environment which might help people learn to manipulate mu power by using complex forms of visual feedback. Perhaps instead of a BreakOut $^{-}$Video game environment, other 3D VR environments would affect learning differently. Using the control steering of a 4-dimensional robot on a terrain might allow subjects to relate better to the task since it would be in a similar context to an activity they commonly perform-namely driving a car. Since the robot's steering is dynamically linked to the relative output of the participant's mu rhythms and is updated in real time, the subject receives the necessary frequent interactions and feedback for efficient learning.

The distributed nature of our specially designed video gaming environment may be used to maintain a level of active engagement, frequent interactions, visually strong feedback, and connections to real-world contexts in order to enhance the learning [21], [30]. On the other hand, the lack of distributiveness in BCI architectures allows only limited visual richness, in which participants receive simple graphical representation of their mu-rhythm output as feedback. The robotic-car like scenario, or any VR environment, would provide an environment that is more interactive, realistic and motivationally engaging and have enough visual cues activity, so that the subject can easily get involved and engaged in the task. This is only achievable through a high processing distributed architecture like the one proposed in this study whereas active engagement is missing from the traditional experiments of mu training and might be a reason 
TABLE II

EACH SUBJECT'S INDIVIDUAL DATA, TRIALS, NO. OF HITS MADE, FAILED TO HIT, NO. OF ABORTS, NO. OF CORRECT MOVEMENT TO THE RIGHT (CMR), NO. of Correct Movement to the Left (CML), NO. OF Wrong Movement to the Right (WMR) AND NO. OF Wrong Movement TO THE LEFT (WML).

(a) Subject A's Results

\begin{tabular}{c|c|c|c|c|c|c|c|c|c}
\hline \hline No. & Trial & Hit & Failed & Abort & CMR & CML & WMR & WML & Rate \\
\hline 1 & 20 & 7 & 2 & 11 & 537 & 823 & 596 & 689 & 77 \\
\hline 2 & 20 & 11 & 0 & 9 & 404 & 842 & 407 & 803 & 100 \\
\hline 3 & 20 & 12 & 2 & 5 & 417 & 756 & 436 & 673 & 80 \\
\hline 4 & 20 & 14 & 0 & 6 & 488 & 721 & 493 & 662 & 100 \\
\hline Sum & 80 & 44 & 4 & 31 & 1846 & 3142 & 1932 & 2827 & $89.25 \%$ \\
\hline \hline
\end{tabular}

(b) Subject B's Results

\begin{tabular}{c|c|c|c|c|c|c|c|c|c}
\hline \hline No. & Trial & Hit & Failed & Abort & CMR & CML & WMR & WML & Rate \\
\hline 1 & 20 & 7 & 6 & 7 & 769 & 497 & 799 & 484 & 53 \\
\hline 2 & 20 & 8 & 5 & 5 & 693 & 282 & 787 & 241 & 53 \\
\hline 3 & 20 & 9 & 4 & 4 & 785 & 369 & 823 & 359 & 56 \\
\hline 4 & 20 & 8 & 5 & 5 & 783 & 317 & 834 & 278 & 53 \\
\hline Sum & 80 & 32 & 20 & 21 & 3030 & 1465 & 3243 & 1362 & $53.75 \%$ \\
\hline \hline
\end{tabular}

(c) Subject C's Results

\begin{tabular}{c|c|c|c|c|c|c|c|c|c}
\hline \hline No. & Trial & Hit & Failed & Abort & CMR & CML & WMR & WML & Rate \\
\hline 1 & 20 & 6 & 6 & 8 & 686 & 445 & 873 & 339 & 50 \\
\hline 2 & 20 & 7 & 7 & 6 & 817 & 401 & 869 & 386 & 50 \\
\hline 3 & 20 & 9 & 7 & 4 & 828 & 421 & 883 & 405 & 56 \\
\hline 4 & 20 & 9 & 9 & 2 & 713 & 234 & 760 & 220 & 50 \\
\hline 5 & 20 & 7 & 7 & 6 & 822 & 414 & 855 & 405 & 50 \\
\hline Sum & 100 & 38 & 36 & 26 & 3869 & 1915 & 4240 & 1755 & $51.2 \%$ \\
\hline \hline
\end{tabular}

(d) Subject D's Results

\begin{tabular}{c|c|c|c|c|c|c|c|c|c}
\hline \hline No. & Trial & Hit & Failed & Abort & CMR & CML & WMR & WML & Rate \\
\hline 1 & 20 & 17 & 2 & 1 & 490 & 511 & 566 & 466 & 89 \\
\hline 2 & 20 & 9 & 10 & 1 & 654 & 166 & 735 & 113 & 47 \\
\hline 3 & 20 & 10 & 10 & 0 & 666 & 133 & 712 & 124 & 50 \\
\hline 4 & 20 & 10 & 7 & 3 & 625 & 251 & 681 & 237 & 58 \\
\hline Sum & 80 & 46 & 29 & 5 & 2435 & 1061 & 2694 & 940 & $61 \%$ \\
\hline \hline
\end{tabular}

why training in those tasks takes a considerable amount of time and less accuracy of control over the targets.

\section{ACKNOWLEDGMENTS}

This project was partially funded by a University of Sydney's Bridging grant. A video of the BCI system is available at http://www.ee.usyd.edu.au/projects/penso.

\section{REFERENCES}

[1] J. R. Millan, F. Renkens, J. Mourino, and W. Gerstner, "Noninvasive brain-actuated control of a mobile robot by human eeg." IEEE Transactions on Biomedical Engineering, pp. 1026-1033, 2004.

[2] G. Pfurtscheller and et. al., "15 years of bci research at graz university of technology: current projects," IEEE Trans. on Neural Systems and Rehabilitation Engineering, pp. 205-210, 2006.

[3] A. Kostov and M. Polak, "Parallel man-machine training in development of eeg-based cursorcontrol," IEEE Trans. on Neural Systems and Rehabilitation Engineering, pp. 203-205, 2000.

[4] J. R. Wolpaw and et. al., "Brain-computer interfaces for communication and control," Clinical Neurophysiology, pp. 767-791, 2002.
[5] M. A. Lebedev, J. M. Carmena, J. E. O’Doherty, M. Zacksenhouse, C. S. Henriquez, J. C. Principe, and M. A. Nicolelis, "Cortical ensemble adaptation to represent velocity of an artificial actuator controlled by a brainmachine interface," Neuroscience, vol. 25(19), pp. 4681-4693, 2005 .

[6] A. Searle and L. Kirkup, "A direct comparison of wet, dry and insulating bioelectric recording electrodes," Physiol. Meas., vol. 21, pp. 271-283, 2000 .

[7] G. Gargiulo, P. Bifulco, R. A. Calvo, M. Cesarelli, A. Fratini, C. Jin, and A. van Schaik, "A wearable dry-electrode-capable bluetooth personal monitoring system," in 4th European Congress for Medical and Biomedical Engineering, Antwerp, Belgium, 23-27 Nov, 2008.

[8] "Emotiv systems." Website, Aug, 2008, http://emotiv.com.

[9] F. Lotte, M. Congedo, A. Lcuyer, F. Lamarche, and B. Arnaldi, "A review of classification algorithms for eeg-based brain-computer interfaces," Journal of Neural Engineering, vol. 4, 2007.

[10] T.-P. Jung, C. Humphries, T.-W. Lee, S. Makeig, M. McKeown, V. Iragui, and T. Sejnowski, "Removing electroencephalographic artifacts: comparison between icaand pca," in IEEE Signal Processing Society Workshop on Neural Networks for Signal Processing VIII, Cambridge, U.K, 1998.

[11] T. P. Jung, S. Makeig, and et. al., "Removing electroencephalographic 
artifacts by blind source separation," Psychophysiol., vol. 37, pp. 168178., 2000.

[12] C. W. Anderson, J. N. Knight, and et. al., "Geometric subspace methods and time-delay embedding for eeg artifact removal and classification," IEEE Transactions on Neural Systems and Rehabilitation Engineering, vol. 14(2), pp. 142-146, 2006.

[13] M. Kubat, I. Koprinska, and et. al., "Learning to classify biomedical signals," Machine Learning, Data Mining, and Knowledge Discovery, 1997.

[14] D. J. McFarland, C. W. Anderson, and et. al., "Bci meeting 2005 workshop on bci signal processing: Feature extraction and translation," IEEE Transactions on Neural Systems and Rehabilitation Engineering, vol. 14(2), pp. 135-138, 2006

[15] O. AlZoubi, I. Koprinska, and R. A. Calvo, "Classification of braincomputer interface data," in AusDm08, The Australasian Data Mining Conference: AusDM 2008 Stamford Grand, Glenelg, Adelaide, 27-28 November 2008.

[16] G. Schalk and et. al., "Bci2000: A general-purpose brain-computer interface (bci) system," IEEE Trans. on Biomedical Engineering, pp. 1034-1043, 2004.

[17] J. W. Kozelka and T. A. Pedley, "Beta-rhythms and mu-rhythms," Journal of Clinical Neurophysiology, pp. 191-207, 1990.

[18] D. J. McFarland and et al., "Mu and beta rhythm topographies during motor imagery and actual movements," Brain Topography, pp. 177$186,2000$.

[19] R. Shadmehr and F. A. Mussa-Ivaldi, "Adaptive representation of dynamics during learning of a motor task," Journal of Neuroscience., vol. 14(5), pp. 3208-3224, 1994.

[20] T. Gulrez, A. Tognetti, A. Fishbach, S. Acosta, C. Scharver, D. De Rossi, and F. A. Mussa-Ivaldi, "Controlling wheelchairs by body motions: A learning framework for the adaptive remapping of space," in International Conference on Cognitive Systems (CogSys 2008), Karlsruhe, Germany, April 2-4, 2008.
[21] T. Gulrez, M. Kavakli, and A. Tognetti, "Robotics and virtual reality: A marriage of two diverse streams of science," in Computational Intelligence in Multimedia Processing: Recent Advances. SpringerVerlag, Hiedelberg-Germany, 2008, pp. 99-118.

[22] D. J. McFarland and J. R. Wolpaw, "Egg-based communication and control: Speed accuracy relationships," Applied Psychophysiology and Biofeedback, vol. (28)3, pp. 217-231, September 2003, http://dx.doi. org/10.1023/A:1024685214655.

[23] “Core system architecture," Website, Aug, 2008, http://www.bluetooth. com/Bluetooth/Technology/Works/Core_System_Architecture.htm.

[24] "Bluetooth radio," Website, Aug, 2008, http://www.palowireless.com/ infotooth/tutorial/radio.asp.

[25] "Oem bluetooth module," Website, Aug, 2008, http://www. corscience.de/en/medical-engineering/oem-solutions/oem-modules/ oem-bluetooth-module-bluesense-ad.html.

[26] "3com wireless bluetooth usb adapter," Website, Aug, 2008, http: //www.3com.com/products/en_US/detail.jsp?tab=support\&pathtype $=$ support\&sku=3CREB96.

[27] "Bci 2000." Website, Aug, 2008, http://www.bci2000.org/BCI2000/ Home.html.

[28] A. Schloegl, K. Lugger, and G. Pfurtscheller, "Using adaptive autoregressive parameters for a brain-computer-interface experiment," in 19th Annual International Conference of the IEEE Engineering in Medicine and Biology Society, Chicago, USA, 1997.

[29] G. Pfurtscheller and et. al., "Graz-bci: state of the art and clinical applications," IEEE Trans. on Neural Systems and Rehabilitation Engineering, pp. 1-4, 2003.

[30] T. Gulrez, M. Kavakli, and A. Tognetti, "Precision position tracking in virtual reality environments using sensor networks," in IEEE International Symposium on Industrial Electronics, Vigo, Spain, 2007. 